American Journal of Applied Sciences 9 (4): 505-509, 2012

ISSN 1546-9239

(C) 2012 Science Publications

\title{
Symbol Timing Estimation in Multiband Orthogonal Frequency Division Multiplexing based Ultrawide band System
}

\author{
Suresh, M.N., S.J. Thiruvengadam and V. Abhaikumar \\ Department of Electronics and Communication Engineering, \\ TIFAC CORE in Wireless Technologies, \\ Thiagarajar College of Engineering, Madurai, 625 015, India
}

\begin{abstract}
Problem statement: The Multiband Orthogonal Frequency Division Multiplexing (MBOFDM) Based Ultra Wideband system (UWB) is highly susceptible to symbol timing error. Symbol timing estimation in MB-OFDM based UWB is difficult due to the presence of strongest path at delayed instant. Approach: Symbol timing estimation has two steps namely a coarse estimation and a fine estimation. In this study, a maximum likelihood framework for the fine timing estimation is proposed. It utilizes the delay embedded in the estimated Channel Impulse Response (CIR) obtained from the cross correlation of training sequence with the received sequence. Results: The performance of the proposed method is analyzed in terms of probability of correct timing detection. It is observed that the proposed technique has better probability of correct timing detection performance compared to the existing algorithm, in all the UWB channel models proposed by IEEE 802.15.3a working group for UWB systems. Conclusion: A novel method to estimate fine symbol timing error for MB-OFDM based UWB system is proposed. This method will be suitable for MB-OFDM receivers for UWB positioning and UWB communication.
\end{abstract}

Key words: Multiband OFDM, symbol timing estimation, signal to interference ratio, UWB channel models

\section{INTRODUCTION}

The Multi-Band Orthogonal Frequency Division Multiplexing (MB-OFDM) has been proposed as a promising technology by IEEE and European Computer Manufacturers Association (ECMA) working groups for short range high data rate UWB communication applications (Saeed et al., 2005; Batra et al., 2004) IEEE Std, 2005. In MB-OFDM based UWB system, the allocated spectrum of $3.1-10.6 \mathrm{GHz}$ by Federal Communication Commission (FCC) is divided into fourteen $528 \mathrm{MHz}$ sub bands. In each sub band data is transmitted using OFDM. The distinct feature of MBOFDM is the use of Zero Padding (ZP) instead of Cyclic Prefix (CP). In a CP based OFDM, there exists a correlation between $\mathrm{CP}$ and OFDM signal. This manifests in the form of ripples in the Power Spectral Density (PSD) of the transmitted signal and hence results in additional power back-off. It has been reported that the use of $\mathrm{ZP}$ has reduced the ripples in PSD to zero (Saeed et al., 2005). In MB-OFDM, the availability of varying channel responses across different sub bands provide diversity gain. However,
MB-OFDM is highly susceptible to timing synchronization errors and causes Inter Carrier Interference (ICI) as in OFDM (Zhou et al., 2007). The first significant multipath component arrives at different delayed instants in different realizations. It causes further complications in the timing estimation.

There exist several preamble-based symbol synchronization methods for MB-OFDM based UWB systems (Yak et al., 2005; Yoon and Chong, 2006; Berger et al., 2006; Li et al., 2008). Yak et al. (2005) proposed a set of heuristic algorithms utilizing the first significant channel tap for timing estimation in ZP MBOFDM. Yoon and Chong (2006) proposed a 'correlation of cross correlation' method to enhance the timing estimation performance. Berger et al. (2006) showed that the timing estimation can be further improved when estimation is performed in two steps, namely coarse synchronization and fine synchronization for CP MB-OFDM. Extended the OFDM synchronization techniques to multiband OFDM. Li et al. (2008) proposed a coarse and fine timing estimation using correlation of training sequences defined in IEEE 802.15.3a standard. It is

Corresponding Author: Suresh, M.N., Department of Electronics and Communication Engineering,

TIFAC CORE in Wireless Technologies, Thiagarajar College of Engineering, Madurai, 625 015, India 
observed that the performance of timing estimation algorithms for ZP MB-OFDM is degraded when the CIR has delayed significant path, as in UWB channels.

In OFDM transmission through multipath fading channel, the problem due to delayed significant path is solved using fine timing estimation which exploits the delay embedded in estimated CIR (Larsson et al., 2001; Zhou and Huang, 2009). The CIR estimation is obtained by performing Inverse Fast Fourier Transform (IFFT) on the frequency domain channel estimate. This requires additional complexity due to channel estimation algorithm and IFFT. Moreover, extension of these algorithms to multiband OFDM is not straight forward because of the presence of colored noise due to overlap add receiver (Batra et al., 2004).

In this study, a fine symbol timing estimation technique is proposed for MB-OFDM based UWB systems utilizing the delay embedded in the CIR. The circular cross correlation of the received sequence with the training sequence is performed to estimate the CIR tentatively. A maximum likelihood framework is developed for fine timing estimation from the CIR. The performance of the proposed algorithm in terms probability of correct timing detection is compared with that of the existing algorithm.

\section{MATERIALS AND METHODS}

System model for multiband OFDM: MB-OFDM system transmits data across multiple sub bands and provides frequency diversity. In MB-OFDM systems dedicated training sequences, called cover codes, are used to achieve timing synchronization in different band groups. Within one band group, the standard preamble consists of 21 packet synchronization sequences, 3 frame synchronization sequences and 6 channel estimation sequences IEEE Std, 2005. OFDM symbol in each band consists of $L$ data subcarriers, $G$ number of zero guard samples with $G=G_{\text {pre }}+G_{\text {suf }}$, where $G_{\text {suf }}$ and $G_{\text {pre }}$ are number of zeros appended as suffix and prefix of the OFDM symbol to overcome the multipath Inter Symbol Interference (ISI) and to allow sufficient time for the switching of oscillators between multiple bands respectively.

The low-pass-equivalent time-domain received vector in the $\mathrm{i}^{\text {th }}$ band of UWB system is given by Eq. 1 :

$y_{i}^{\prime}=x_{i}^{\prime} * h_{i}+w_{i}^{\prime} ; 1 \leq i \leq N_{B}$

Where:

$\mathrm{x}_{\mathrm{i}}^{\prime}=$ The $(\mathrm{L}+\mathrm{G}) \times 1$ vector consisting of $\mathrm{L}$ transmitted training sequence appended with $\mathrm{G}$ zeroes $\mathrm{h}_{\mathrm{i}}=$ The $\mathrm{C} \times 1$ channel impulse response

$* \quad=$ Represents convolution

Discarding the last $\mathrm{C}$ number of zeros in the linear convolution, $y_{i}$ is a $(L+G) \times 1$ vector and $w_{i}$ is the $(\mathrm{L}+\mathrm{G}) \times 1$ noise vector with variance $\mathrm{I}_{\mathrm{L}+\mathrm{G}} . \mathrm{N}_{\mathrm{B}}$ is the number of sub bands.

The initial operation in a MB-OFDM receiver is to determine the start of the OFDM symbol in a specific band. This is performed in two steps, namely coarse synchronization and fine synchronization. The coarse synchronization is performed using a correlator to yield an initial timing estimate ( $\mathrm{Li}$ et al., 2008). It provides an approximate estimate of the start of the OFDM symbol in a band.

Consider $\left(\mathrm{L}+\mathrm{G}_{\text {suf }}\right) \times 1$ received signal vector chosen from the start of the OFDM symbol specified by coarse synchronization. This vector is considered to be having a timing error of ' $\mathrm{d}$ ' samples. A simple model with the timing error is developed by adding the last $\mathrm{G}_{\text {suf }}$ number of samples with the first $G_{\text {suf }}$ number of samples of the received vector. The resulting $\mathrm{L} \times 1$ vector in $\mathrm{i}^{\text {th }}$ band with the timing error of ' $\mathrm{d}$ ' is represented as Eq. 2:

$\mathrm{y}_{\mathrm{i}}=\mathrm{XJ}^{\mathrm{d}} \mathrm{Th}_{\mathrm{i}}+\mathrm{w}_{\mathrm{i}} ; 1 \leq \mathrm{i} \leq \mathrm{N}_{\mathrm{B}}$

Where:

$\mathrm{X}=\mathrm{A} \quad \mathrm{L} \times \mathrm{L}$ circulant matrix of training samples

$J=\left[\begin{array}{cc}0_{1 \times(L-1)} & 1 \\ I_{(L-1)} & 0_{(L-1) \times 1}\end{array}\right]=$ The $\mathrm{L} \times \mathrm{L}$ circular shift matrix

$\mathrm{T}=\left[\begin{array}{c}\mathrm{I}_{\mathrm{C}} \\ \mathrm{O}_{(\mathrm{L}-\mathrm{C}) \times \mathrm{C}}\end{array}\right] \quad=$ The $\mathrm{L} \times \mathrm{C}$ tail zero insertion matrix

$J^{\mathrm{d}} T h_{i}$ represents the circular shift of $h_{i}$ with delay $d$ and $\mathrm{XJ}^{\mathrm{d}} \mathrm{Th}_{\mathrm{i}}$ is the circular convolution between training samples and delayed impulse response. $\mathrm{w}_{\mathrm{i}}$ is the colored noise in $i^{\text {th }}$ band with a covariance matrix $R_{w w}^{i}$. The noise is colored due to overlap and add operation.

The circular cross correlation of the received vector $\mathrm{y}_{\mathrm{i}}$ with the training sequence is given by Eq. 3 :

$\mathrm{X}^{\mathrm{H}} \mathrm{y}_{\mathrm{i}}=\mathrm{X}^{\mathrm{H}} \mathrm{XJ}^{\mathrm{d}} \mathrm{Th}_{\mathrm{i}}+\mathrm{X}^{\mathrm{H}} \mathrm{w}_{\mathrm{i}}$

The training sequence is approximately flat in spectrum (Batra et al., 2004) and $\mathrm{X}^{\mathrm{H}} \mathrm{X}$ can be approximated as $\mathrm{LI}_{\mathrm{L}}$. Hence (2) can be represented as Eq. 4: 
$\mathrm{X}^{\mathrm{H}} \mathrm{y}_{\mathrm{i}}=\mathrm{LI}_{\mathrm{L}} \mathrm{J}^{\mathrm{d}} \mathrm{Th}_{\mathrm{i}}+\mathrm{X}^{\mathrm{H}} \mathrm{w}_{\mathrm{i}}$

The term $\mathrm{X}^{\mathrm{H}} \mathrm{w}_{\mathrm{i}}$ in (4) is the colored noise with covariance matrix $\mathrm{XR}_{\mathrm{ww}}^{\mathrm{i}} \mathrm{X}^{\mathrm{H}}$. Though (4) appears to be traditional cross correlation it gives knowledge about the delay embedded in the channel impulse response.

The estimate of channel impulse response with a timing error of ' $\mathrm{d}$ ' is given by Eq. 5 :

$\hat{\mathrm{h}}_{\mathrm{i}}^{\mathrm{d}}=\frac{1}{\mathrm{~L}} \mathrm{X}^{\mathrm{H}} \mathrm{y}_{\mathrm{i}}=\mathrm{J}^{\mathrm{d}} T \mathrm{~h}_{\mathrm{i}}+\tilde{\mathrm{w}}_{\mathrm{i}}$

where, $\tilde{\mathrm{w}}_{\mathrm{i}}$ is colored noise with covariance $\tilde{\mathrm{R}}_{\mathrm{ww}}^{\mathrm{i}}=\frac{1}{\mathrm{~L}^{2}} \mathrm{XR}_{\mathrm{ww}}^{\mathrm{i}} \mathrm{X}^{\mathrm{H}}$.

Maximum likelihood estimation of fine symbol timing: Assuming that the channel coefficients of each band are uncorrelated and $\mathrm{d}$ as deterministic unknown, the estimate of the channel impulse response vector $\hat{h}_{i}^{d}$ is Gaussian distributed with mean zero and covariance matrix $\tilde{R}_{\text {hh }}^{i}$. The likelihood function for the estimation of $d$ is given by Eq. 6 and 7:

$$
\begin{aligned}
& \Lambda\left(\hat{\mathrm{h}}_{1}^{\mathrm{d}}, \ldots, \hat{\mathrm{h}}_{\mathrm{N}_{\mathrm{B}}}^{\mathrm{d}} ; \mathrm{d}\right)=\left(\prod_{\mathrm{i}=1}^{\mathrm{N}_{\mathrm{B}}} \frac{1}{\pi \operatorname{det}\left(\tilde{\mathrm{R}}_{\mathrm{hh}}^{\mathrm{i}}\right)}\right) \\
& \exp \left\{-\sum_{\mathrm{i}=1}^{\mathrm{N}_{\mathrm{B}}}\left(\left(\hat{\mathrm{h}}_{\mathrm{i}}^{\mathrm{d}}\right)^{\mathrm{H}}\left(\tilde{\mathrm{R}}_{\mathrm{hh}}^{\mathrm{i}}\right)^{-1} \hat{\mathrm{h}}_{\mathrm{i}}^{\mathrm{d}}\right)\right\}
\end{aligned}
$$

Where:

$$
\tilde{\mathrm{R}}_{\mathrm{hh}}^{\mathrm{i}}=\mathrm{E}\left(\hat{\mathrm{h}}_{\mathrm{i}}^{\mathrm{d}}\left(\hat{\mathrm{h}}_{\mathrm{i}}^{\mathrm{d}}\right)^{\mathrm{H}}\right)=\mathrm{J}^{\mathrm{d}} \mathrm{TR}_{\mathrm{hh}}^{\mathrm{i}} \mathrm{T}^{\mathrm{H}} \mathrm{J}^{\mathrm{d}}+\frac{1}{\mathrm{~L}^{2}} \mathrm{X} \tilde{\mathrm{R}}_{\mathrm{ww}}^{\mathrm{i}} \mathrm{X}^{\mathrm{H}}
$$

Here, $\mathrm{R}_{\mathrm{hh}}^{\mathrm{i}}$ is the correlation of the $\mathrm{i}^{\text {th }}$ band channel. The $\log$ likelihood function of (6) is Eq. 8:

$$
\begin{aligned}
& \ln \left\{\Lambda\left(\hat{\mathrm{h}}_{1}^{\mathrm{d}}, \ldots, \hat{\mathrm{h}}_{\mathrm{N}_{\mathrm{B}}}^{\mathrm{d}} ; \mathrm{d}\right)\right\}=\ln \left\{\prod_{\mathrm{i}=1}^{\mathrm{N}_{\mathrm{B}}} \frac{1}{\pi \operatorname{det}\left(\tilde{\mathrm{R}}_{\mathrm{hh}}^{\mathrm{i}}\right)}\right\} \\
& -\left\{\sum_{\mathrm{i}=1}^{N_{\mathrm{B}}}\left(\left(\hat{\mathrm{h}}_{\mathrm{i}}^{\mathrm{d}}\right)^{\mathrm{H}}\left(\tilde{\mathrm{R}}_{\mathrm{hh}}^{\mathrm{i}}\right)^{-1} \hat{\mathrm{h}}_{\mathrm{i}}^{\mathrm{d}}\right)\right\}
\end{aligned}
$$

In (7), since UWB channel taps are uncorrelated (Molisch et al., 2003), $\tilde{\mathrm{R}}_{\mathrm{hh}}^{\mathrm{i}}$ is independent of d. Hence considering the second term in (8), the Maximum Likelihood (ML) estimate of delay d is given by Eq. 9:

$$
\hat{\mathrm{d}}=\underset{\mathrm{d}}{\arg \min }\left\{\sum_{\mathrm{i}=1}^{\mathrm{N}_{\mathrm{B}}}\left(\left(\hat{\mathrm{h}}_{\mathrm{i}}^{\mathrm{d}}\right)^{\mathrm{H}}\left(\tilde{\mathrm{R}}_{\mathrm{hh}}^{\mathrm{i}}\right)^{-1} \hat{\mathrm{h}}_{\mathrm{i}}^{\mathrm{d}}\right)\right\}
$$

The estimation of ' $\mathrm{d}$ ' is computationally complex due to $\left(\tilde{\mathrm{R}}_{\mathrm{hh}}^{\mathrm{i}}\right)^{-1}$. The close observation of $\tilde{\mathrm{R}}_{\mathrm{hh}}^{\mathrm{i}}$ reveals that it is diagonally dominant. For a diagonally dominant auto correlation matrix, the matrix inversion can be approximated by inverting its diagonal elements (Molisch et al., 2003). Considering diagonal elements of $\tilde{\mathrm{R}}_{\mathrm{hh}}^{\mathrm{i}}$ as $\left\{\tilde{\mathrm{r}}_{\mathrm{i} 0}, \tilde{\mathrm{r}}_{\mathrm{i} 1} \ldots \tilde{\mathrm{r}}_{\mathrm{i}(\mathrm{L}-1)}\right\}$ and $\hat{\mathrm{h}}_{1}^{\mathrm{d}}=\left[\tilde{\mathrm{h}}_{\mathrm{i} 0}, \tilde{\mathrm{h}}_{\mathrm{i} 1} \ldots \tilde{\mathrm{h}}_{\mathrm{i}(\mathrm{L}-1)}\right]^{\mathrm{T}}$, the optimal estimate of 'd' for the MB-OFDM based UWB system is given by Eq. 10:

$$
\hat{\mathrm{d}}=\underset{\mathrm{d}}{\arg \min }\left\{\sum_{\mathrm{i}=1}^{\mathrm{N}_{\mathrm{B}}} \sum_{\mathrm{l}=\mathrm{d}}^{\mathrm{d}+\mathrm{C}-1} \frac{\left|\tilde{\mathrm{h}}_{\mathrm{il}}\right|^{2}}{\tilde{\mathrm{r}}_{\mathrm{il}}}\right\}
$$

\section{RESULTS}

Mante Carlo simulation is carried out in MATLAB to evaluate the performance of the proposed algorithm and the SIR analysis. The probability of correct timing detection is considered for performance analysis. The simulation parameters are as given in Table 1.

The performance of the proposed algorithm is compared with the method in (Li et al., 2008) where in the fine timing is estimated by finding the timing instant at which maximum of timing metric occurs. The timing metric is a sum of magnitude of autocorrelation of the received sequences in three consecutive bands. The additional parameters required to simulate the method in (Li et al., 2008) are correlation window length and the correction constant term $\eta$. The value of $\eta$ depends on the type of channel. For CM1 model it is chosen to be 4.The correlation window length is chosen to be 128 .

\begin{tabular}{ll} 
Table 1: Simulation parameters & \\
\hline No. of Subcarriers, $\mathrm{L}$ & 128 \\
Length of suffix, $\mathrm{G}_{\text {suf }}$ & 32 \\
Length of prefix, $\mathrm{G}_{\mathrm{pre}}$ & 5 \\
Training sequence & TFC1 (Batra et al., 2004) \\
Channel model & CM1, CM2, CM3 and \\
& CM4 (Molisch et al., 2003) \\
Number of UWB realizations & 100 \\
\hline
\end{tabular}

Table 1: Simulation parameters

Length of suffix, $\mathrm{G}_{\text {suf }}$

Length of prefix, $\mathrm{G}_{\text {pre }}$

Training sequence TFC1 (Batra et al., 2004)

Number of UWB realizations 


\section{DISCUSSION}

Figure 1 shows the probability of correct timing detection of the proposed algorithm in CM1 channel by varying the number of bands considered for detecting the fine timing error. It is observed that the probability of detection increases exponentially at low SNR region and reaches a constant at high SNR region. The SNR required to attain a detection probability of 0.9 is 12.2 $\mathrm{dB}$ when the number of bands is one. However the same is attained for a SNR of 9.4 and $8.6 \mathrm{~dB}$ when the number of bands is two and three respectively. The exponential gain in the SNR as the number of bands are increased, is similar to the gain obtained in detection of data transmitted through fading channels with diversity. Hence the improvement in the probability of correct timing detection is due to the inherent frequency diversity in MB-OFDM. This reasoning is made, since the channel impulse response in each band are independent, there is a possibility that the first path in any one of the bands may not be in deep fade. Further, It is noted that the proposed algorithm with single band provides a SNR gain of $10 \mathrm{~dB}$ for a detection probability of 0.6 when compared to method in $(\mathrm{Li}$ et al., 2008). This performance improvement is due to the usage of delay embedded in CIR.

Figure 2-4 show the detection performance of the proposed algorithm in CM2, CM3 and CM4 channel models respectively. For CM2, CM3 and CM4 channel models, the value of $\eta$ is chosen to be 8,9 and 14 respectively. Similar to the performance in CM1, the detection performances in CM2, CM3 and CM4 channel models show improvement with number of bands.

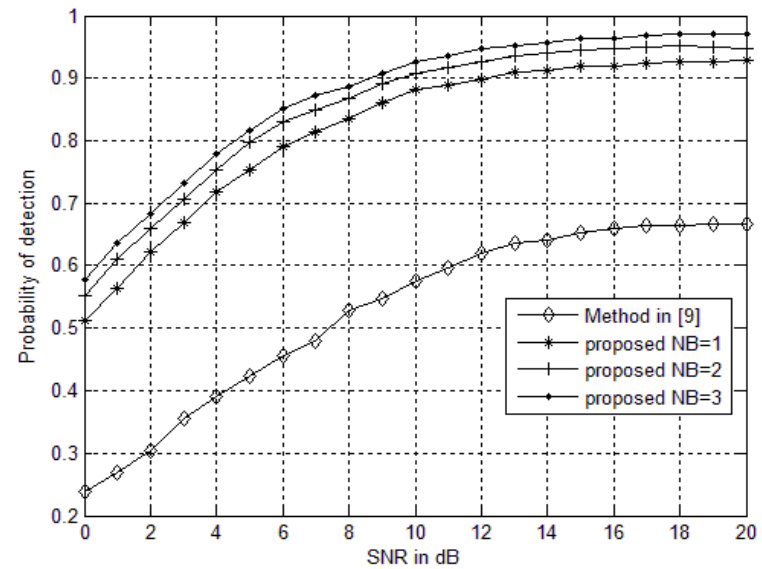

Fig. 1: Probability of detection of proposed algorithm in CM1channel model

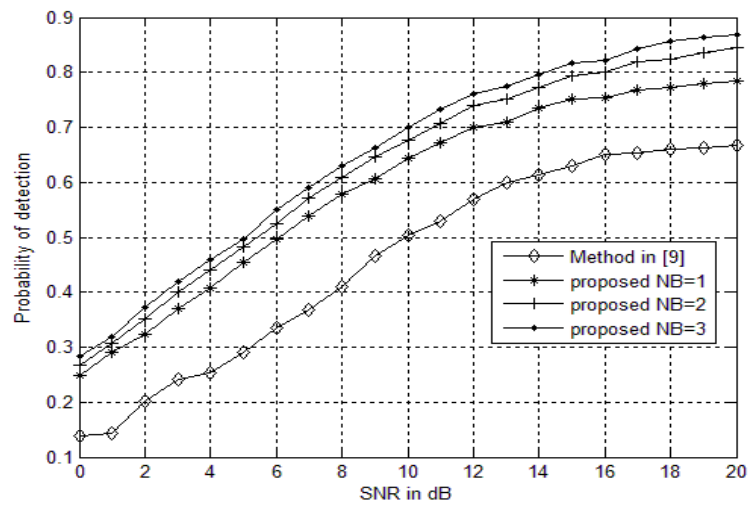

Fig. 2: Probability of detection of proposed algorithm in CM2 channel model

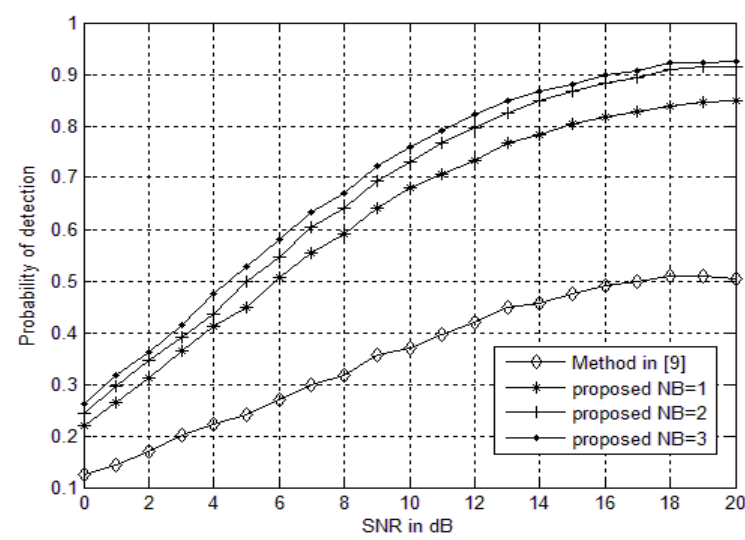

Fig. 3: Probability of detection of proposed algorithm in CM3 channel model

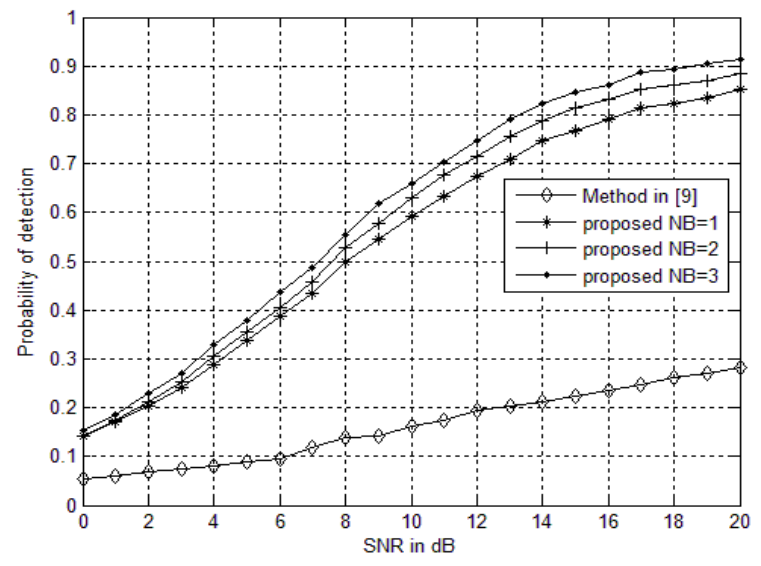

Fig. 4: Probability of detection of proposed algorithm in CM4 channel model 
Am. J. Applied Sci., 9 (4): 505-509, 2012

\section{CONCLUSION}

In this study, a preamble based novel fine symbol timing estimation technique for MB-OFDM based UWB systems is proposed. It is observed that the proposed algorithm provides significant improvement in probability of correct timing detection when compared to existing method. It is also observed that the timing performance increases with the number of bands due to inherent frequency diversity.

\section{ACKNOWLEDGMENT}

The researchers are very much thankful to the Management of Thiagarajar College of Engineering for their support and assistance to carry out this study.

\section{REFERENCES}

Batra, A., J. Balakrishnan, G.R. Aiello, J.R. Foerster and A. Dabak, 2004. Design of a multiband OFDM system for realistic UWB channel environments. IEEE Trans. Microwave Theory Technol., 52: 2123-2138. DOI: 10.1109/TMTT.2004.834184

Berger, C.R., S. Zhou, Z. Tian and P. Willett, 2006. Precise timing for multiband OFDM in a UWB system. Proceedings of the IEEE International Conference on Ultra-Wideband, Sept. 24-27, IEEE Xplore Press, Waltham, MA., pp: 269-274. DOI: 10.1109/ICU.2006.281561

Li, Y., H. Minn and R.M.A. Rajatheva, 2008. Synchronization, channel estimation and equalization in MB-OFDM systems. IEEE Trans. Wireless Commun., 7: 4341-4352. DOI: 10.1109/T-WC.2008.070601
Larsson, E.G., G. Liu, J. Li and G.B. Giannakis, 2001. Joint symbol timing and channel estimation for OFDM based WLANs. IEEE Commun. Lett., 5: 325-327. DOI: 10.1109/4234.940980

Molisch, A.F., J.R. Foerster and M. Pendergrass, 2003. Channel models for ultrawideband personal area networks. IEEE Wireless Commun., 10: 14-21. DOI: 10.1109/MWC.2003.1265848

Saeed, R., S. Khatun, B.M. Ali and M.K. Abduallah, 2005. Ultra Wide Band (UWB) ad-hoc networks: review and trends. J. Comput. Sci., 1: 35-39. DOI: 10.3844/jcssp.2005.35.39

Yak, C.W., Z. Lei, S. Chattong and T.T. Tjhung, 2005. Timing synchronization and frequency offset estimation for Ultra-Wideband (UWB) Multi-Band OFDM systems. Proceedings of the IEEE 16th International Symposium on Personal, Indoor and Mobile Radio Communications, Sept. 11-14, IEEE Xplore Press, Berlin, pp: 471-475. DOI: 10.1109/PIMRC.2005.1651481

Yoon, S.H. and J.W. Chong, 2006. Packet detection and symbol timing synchronization algorithm for multiband OFDM UWB. IEICE Trans. Commun., 89: 1433-1435.

Zhou, Y., A.I. Karsilayan and E. Serpedin, 2007. Sensitivity of multiband ZP-OFDM ultra-wideband and receivers to synchronization errors. IEEE Trans. Signal Process., 55: 729-734. DOI: 10.1109/TSP.2006.885762

Zhou, H. and Y.F. Huang, 2009. A maximum likelihood fine timing estimation for wireless OFDM systems. IEEE Trans. Broadcast., 55: 3141. DOI: $10.1109 /$ TBC.2008.2007457 